\title{
SETI AND SPACE LAW: JURISPRUDENTIAL AND PHILOSOPHICAL CONSIDERATIONS FOR HUMANKIND IN RELATION TO EXTRATERRESTRIAL LIFE $\dagger$
}

\author{
PATRICIA M. STERNS \\ Law Offices of Sterns and Tennen, Attorneys and Counselors at Law, Phoenix, Arizona, USA
}

\begin{abstract}
The impact of confirmation of life outside the small ecosphere we call Earth will be profound on the terran population as a whole. The "Declaration Of Principles Concerning Activities Following The Detection Of Extraterrestrial Intelligence" and the IAA Position Paper "A Decision Process for Examining the Possibility of Sending Communications To Extraterrestrial Civilizations: A Proposal" provide a firm basis for the development of a new body of space law. It is important that space law design and prepare for implementation of a protocol to guide the nations of the world concerning the search for extraterrestrial intelligence (SETI), through the advice and cooperation of scientists, jurisprudential, philosophical, political and sociological scholars. Through the IAA, the IISL, the United Nations and other organizations, formal documentation should be drafted to encode the Declaration of Principles and IAA Position Paper referred to above. In this way, a body of metalaw can be developed to enable human communication with non-terrestrial life. This paper discusses the philosophical and sociological parameters of terran understanding of our place in the universe which will dramatically impact jurisprudential thought and action in light of the realization of the infinitesimally small niche that humankind occupies. A discussion of these interdisciplinary concerns will be necessary to realize a metalegal approach to interstellar communications and relations. (C) 2000 Elsevier Science Ltd. All rights reserved
\end{abstract}

\section{INTRODUCTION}

The search for extraterrestrial intelligence (ETI) presents one of the most fundamental questions formulated by mankind: are we alone in the universe? The answer to this question is basic to our understanding of our place in the cosmos, its origin and its destiny [1]. For millennia, many religious beliefs placed mankind at the center of the universe, both physically and spiritually. The secular approach, and some more recent religious interpretations, however, have focused on the presence of intelligent life in this modest corner of an otherwise unremarkable galaxy, and thereby reasoned that life must be plentiful on a wide range of otherwise mediocre planets. This "principle of mediocrity may appear to express modesty and even humility. By making intelligence a natural and inevitable feature of life in the universe, it raises the quality most prized by humans to a norm of the universe" [2].

It has been posited that the detection of ETI would complete the Copernican revolution. That is, "[i]n spite of the fact that Copernicus showed that the Earth was not the center of the universe, man has continued to believe that even though he was

$\dagger$ IAF Paper No. IAA-96-IAA.9.2.08 presented at the 47th International Astronautical Congress at the 25th Review Meeting of the Search for Extraterrestrial Intelligence. not geographically in the center of the universe, he is central in essence. This common belief would be shattered in the presence of ETI" [3]. There can be no doubt that the detection of ETI would be a profound experience for the human species, and present issues and questions crossing all cultural, political, economic, jurisprudential and philosophical lines. There also can be no doubt that the impact of detection requires detailed and thoughtful consideration, which avoids both simplistic and unsupported conclusions. Furthermore, the cultural, political, economic, jurisprudential and philosophical beliefs of the ETI will affect terran understanding of the contact.

\section{POLICY IMPLICATIONS OF DETECTION}

The discussion of the impact the detection of ETI would have on society assumes that information concerning the detection will be released to the general public. This information extends to both the fact of detection, as well as the substance, if any, of the contact. The Declaration of Principles Concerning Activities Following the Detection of Extraterrestrial Intelligence mandates such dissemination of information about detection of an ETI [4]. However, compliance with this Declaration may not be forthcoming, as political reasons and other factors may militate against the disclosure of any information concerning the existence of or 
communication with ETI. Thus, the first policy issue to be presented by confirmation of contact with an ETI is whether the information should be released to the public [5].

Reijnen has noted that SETI experiments primarily have been under the control of national governments, and that municipal law will control the exchange and dissemination of information domestically and internationally for national political reasons. "Governments could attempt to control or monopolize the information in efforts to maximize their political benefit. Significant partisan political consequences could result from successful or unsuccessful management of the information that accompanies detection" [2,4-2]. The potential for military, commercial or political advantage by the use of information in a confirmed signal may provide motivation to a government to deny dissemination of the information even to its own scientific community [7].

A state may be reluctant to disclose news of confirmed detection of ETI for fear of triggering widespread panic. The impact of detection has been predicted to be in a range from "catastrophic" to "millennial" [2, p. 2-16-2-17], from paranoia to "pronoia" [8]. At one extreme, the impact could result in anomie, that is, the sudden lack of rules with a concomitant authority crisis, resulting in the disintegration of society [3]. At the other end of the spectrum is the view that the news of the presence of ETI would be greeted with widespread interest but relative apathy by the general public. However, the consensus of opinion is that the impact of detection would be profound on society, culture and philosophy $[1$, p.194, 9,10]. In either case, perhaps the only conclusion which can be drawn at this time is that from the moment of contact on, there will be no way back to isolation [2, p. 2-18,9, p. 28].

Fears of widespread panic on the release of news of contact with ETI may be overestimated. As stated by Ashkenazi, "the human response to CETI would consist of nothing and of much: two weeks of ado replaced by disinterest as a newer item hits the media, and a far reaching, unpredictable change in human thinking, and thus, ultimately, behaviors". The recent report of possible life on Mars is a case in point. The world watched with great interest as NASA announced the discovery of possible fossilized bacteria on a meteorite believed to have originated from Mars [12]. However, the hysteria which some might have expected did not occur. Most people seemed to accept the discovery as confirmation of what they professed they had always believed. Perhaps discovery of an ETI will result in a similar level of interest and benign acceptance.

$\dagger$ It has been noted that the issues of whether and what to respond are essentially the same as those raised by proposals to send messages to attract detection.
However, the existence of a living, intelligent being carries further and more serious implications than possible fossilized carbon remains [1, p. 195].

A structure must be developed for international and transnational cooperation to formulate an appropriate response to the news of contact with ETI. The maintenance of trust and credible relations between the government, the public and educational, scientific and other institutions is essential to reduce fears and suspicions that will accompany any announcement of contact with an ETI [2, p. I-2]. The Draft Declaration of Principles Concerning the Sending of Communications to Extraterrestrial Intelligence, contained in the IAA Position Paper, calls for the initiation of international consultations to determine whether a reply to a detected communication should be made, and if so, the content of such a reply [10, Annex 2]. This approach has received widespread support among the commentators [6,13-15], and underscores the policy considerations at both the national and international levels that should be included in a future international agreement [5]. The Draft Declaration of Principles has been criticized, however, as an impingement on national sovereignty by restricting the right of individual states to act and react to events which would stand among the most momentous in history. Nevertheless, the limitations placed on sovereignty by the Declaration are no more significant than that presented by any international agreement in general.

The obligation to disclose the discovery of organic life found on the Moon or elsewhere, such as provided in the Moon Treaty [16,17], should be clarified and strengthened by the conclusion of a treaty expressly requiring the public release and disclosure of both the fact and content of a detected signal or other discovery of ETI within a specified period of time following verification. The agreement also should provide for the international protection of the electromagnetic frequency on which an extraterrestrial signal is transmitted to protect and preserve the frequency band for further search and research $[2,4,8,6,18]$.

\section{POLICY IMPLICATIONS OF A REPLY}

The next significant policy issue is: should a response be sent to the signal $[5,8,9,10,2] . \dagger$ This question may largely be academic and moot. Humankind does not exist in electromagnetic secrecy. Communication by radio signal fundamentally is a two-way process. While we are scanning the sky for possible signals emanating from ETI, we also are transmitting signals which could be received by an ETI. Broadcasts and transmissions by means of radio waves, TV carrier waves, radar transmissions and microwaves have been conducted for more than 50 years. Thus, a sphere more than 100 light years in diameter has been created which has 
been reached by terrestrial broadcasts. This sphere encompasses approx. 10,000 stars [2,1-4]. Unintentional communications to ETI from Earth have been supplemented with transmissions and messages specifically intended for an extraterrestrial recipient, such as the Arecibo message, and the Voyager and Pioneer information packages [18, pp 19-26]. The possibility, therefore, must be considered that the first contact with ETI confirmed by humankind is itself a response to a signal or artifact originating from this planet. $\dagger$

The substance and content of any response to a confirmed signal from an ETI will involve numerous considerations of policy, philosophy and jurisprudence. The nature of the detection will have a direct impact on the substance of the response. An electromagnetic signal, for example, could be merely a call, devoid of any substantive information itself [15]. Alternatively, the signal could be intended to attract the attention of another intelligent life form, and possibly even be directed to Earth [10, 2]. A detected signal also could be our serendipitous reception of an internal communication of another civilization $[10,2]$, or communication between two other intelligent life forms on different planets. Each circumstance would be unique, and the corresponding response would need to be unique. Furthermore, a premeditated contact may require a more rapid reply than a stray contact [19]. Heidmann has observed

If we look beyond the short span of a single human being's lifetime, it is not unreasonable to envisage interstellar dialogs [sic] that outlast it by a wide margin... If we want to start a dialog [sic], we shall, according to Goldsmith, be in a competition with a lot of other 'correspondents' that have already contacted the civilization that is transmitting. A poorly prepared reply will not have much chance of initiating sustained communication. Our initial response may play a decisive part in starting a dialog [sic]; we need to have the most intelligent stock of knowledge to put forward, which could not be done in confusion [1, p. 200].

A response could be as simple as a signal of obvious non-natural origin which carries no substantive message, but would evidence the existence of intelligence, to the alternatively complex transmission of an encyclopedia [1, 200]. Macrea has

$\dagger$ The Voyager spacecraft carried a selection of recordings of music from Earth, ranging from Bach to "Johnny B. Goode" by Chuck Berry. Following the launch of Voyager, an American satirical television show, Saturday Night Live, reported on the detection of the first signal from an ETI. According to SNL, this first message from space was "Send more Chuck Berry".

\#In the event a message were directed to Earth by an ETI, it would be reasonable to consider that the ETI may have intercepted our communications or otherwise studied our society, culture and politics. Thus, an ETI may be able to determine whether the content of a response from Earth was truthful. expressed the policy concerns that the response should neither be too long nor difficult to decode $[20,179]$. The consideration of this issue must include the possibility that more than one message can be sent simultaneously, and that both simple and complex messages may be included in a response. In any event, a reply should be truthful $[21]+$.

\section{POLICY IMPLICATIONS OF METALAW}

The detection of a signal from an ETI will require mankind to prepare "to deal with intelligent beings who are by nature different in kind and who live in environments which are different in kind. Although these propositions open great areas of juridical speculation, it is sufficient at this time to establish the simple proposition that we must forgo any thought of enforcing our legal concepts on other intelligent beings" [22]. Terrestrial nations must realize that any ETI will be beings "with their own understanding of a kind of 'rules of behaviour' and thus, be legal subjects" [13]. Furthermore, in regard to contact between two intelligent races, a basic understanding of "mutual rules will lead to a "code of conduct"" [13]. This is the starting point for metalaw. The ultimate question, however, may be the respective positions of humankind and ETI relative to the origin of life and their views of self, prior to discovery and verification of the other's existence.

It can be asserted that natural moral law transcends the universe, and that any sentient beings will discover the same moral truths which will apply to both intelligent races [2,2-4]. This may, however, be a situation, not where reasonable minds may differ, but where reasoning minds may differ. The ability of humans to reason and think logically is inherently and inexorably intertwined with our environment, biology and evolution. The language used to communicate itself "forms the cultural context within which members of a culture are able to envision, articulate, and imagine things other than themselves" [2,3-10]. Thus, our thought processes may be unique to these circumstances. Similarly, an ETI's ability to think and reason will be derived from its indigenous environment, its unique history and its biology. Therefore, we must consider the possibility that in logic, as in physics, everything may be relative [23].

Morality may influence metalaw in another, equally important context. That is, we cannot approach relations with alien intelligences from a moral void. Specifically, we must consider the moral implications of our actions on an ETI, as well as the implications of their actions on human morality. Two examples will illustrate the matter. The first concerns Haley's classic principle of metalaw, that

We must do unto others as they would have done unto them. To treat others as we would desire to be treated 
might well mean their destruction. We must treat them as they desire to be treated. This is the simply expressed but vastly significant premise of metalaw [22, p. 395]

This principle could present a significant dilemma if the ETI desired us to act in a manner toward them which were repugnant to our moral code and sensibilities. Perhaps, on an alien world, wars of conquest constitute the norm, and further that the victors practice cannibalism or slavery on the vanquished, or require that the vanquished pay homage to the deity or deities of the victors [24]. In such a situation, the ETI may desire for us to engage them in an interplanetary war of conquest, with cannibalism or slavery or religious subjugation as the expected fate of one party.

The second situation concerns circumstances where a race is unaware that its acts are harmful to the other intelligent life. Would a duty exist for the actor to make an inquiry as to whether its actions were causing harm, no matter how inadvertent or unintended. Conversely, would the damaged race be obligated to determine the intent of the actor and/or to seek a peaceful resolution between them. As noted by Haley

In metalaw we can project only one principle of human law, namely the stark concept of absolute equity. The natural law of man, as we know it, is anthropocentric in kind, and while such law must govern in space among human beings, it nevertheless is the law of human nature alone. With the concept of absolute equity, we shall be prepared to face the possibility of an indefinite number of natures and, therefore, of an indefinite number of frameworks of natural laws [22, p. 414].

The temptation to reply to a message received from an ETI may be too great to resist. The prospects of engaging in a dialog with intelligent beings from another part of the universe are too fantastic to dismiss. At the very least, sufficiently sophisticated broadcast equipment is available to various groups and individuals to enable them to send messages, even if such activities are conducted clandestinely or contrary to local laws and regulations. Thus, to avoid renegade reaction, an international agreement or treaty should include appropriate provisions by which a global response can be formulated. Reijnen asserts that the drafting of a reply by the international community "seems to offer the best guarantee that any nationalistic, political or other unfavorable tendency in a return message is avoided" $[14,19]$.

The detection of ETI will not necessarily result in communication with ETI. Whether due to physical, biological, spiritual or other restraints, "the detection of the long-sought signal, might be more frustrating than exhilarating. Unable to understand or to communicate with detected ETI's we would know that, in a profound sense, we are alone in the universe" [2,2-24]. Such a view, however, is unduly pessimistic. It could be that the obstacles to communication will present a new challenge to the human spirit. If an extraterrestrial race is intelligent and communicative, then mankind shall determine a way to communicate with them. Ultimately, this quest, may have a beneficial, unifying effect on human civilization.

\section{CONCLUSION}

A treaty must be drafted to develop a body of metalaw which will enable human communication with and understanding of non-terrestrial intelligent beings. It is axiomatic that the philosophical and sociological parameters of terran understanding of our place in the universe will affect our development of metalaw. Thus, humans must put aside their egocentric, anthropomorphic view of self, and proceed on the assumption that our civilization, like our own star, is like a grain of sand on the beach. We must recognize that the concept of equity shall be extended to all intelligent, sentient beings. The best reply to any signal we may receive may be simply to advise other intelligent beings that intelligent life exists on planet Earth [20].

Acknowledgements - This paper is dedicated in memory of my mother, Mildred D. Sterns, who has passed from this earth, forever to be a light among the stars.

\section{REFERENCES}

1. Heidmann, J., Extraterrestrial Intelligence, 1995, 196.

2. SETI Institute. Social implications of detecting an extraterrestrial civilization. A report of the Workshops on the Cultural Aspects of SETI 2-12, 1994.

3. Pinotti, R., Contact: releasing the news. Acta Astronautica, 1990, 21, 109, 110.

4. Declaration of principles concerning activities following the detection of extraterrestrial intelligence. Acta Astronautica, 1990, 21, 153.

5. Logsdon, J. M. and Anderson, C. M., Announcing the first signal: policy aspects. Acta Astronautica, 1990, 21, 89

6. Reijnen, G. C. M., The nations united in the scientific and political debate in the search for extraterrestrial intelligence 2-3. IAF Paper No. IAA.94-IAA.9.2.734, 1994. If the detection were made by a private organization rather than by a government program, the information might not be in the public domain.

7. Tough, A., Critical examination of factors that might encourage secrecy. Acta Astronautica, 1990, 21, 97.

8. Finney, B., The impact of contact. Acta Astronautica, 1990, 21, 117.

9. Fasan, E., Relations with Alien Intelligences, 1970, 29.

10. IAA Position Paper. A decision process for examining the possibility of sending communications to extraterrestrial civilizations 2-3, 1995.

11. Ashkenazi, M. Not the sons of Adam: religious responses to ETI. IAF Paper No. IAA-91-610, 1991, citing Beck, Extraterrestrial intelligent life. In Extraterrestrials, vol. 3, p. 17 (ed. E. Regis, 1985). Cambridge University Press. 
12. McKay, D. S., et al., Search for past life on Mars: possible relic biogenic activity in martian meteorite ALH84001. Science, 1996, 273, 924.

13. Fasan, E., Legal consequences of SETI detection, IAF Paper No. IAA-95-IAA 9.2.04, 1995.

14. Michaud, M., A reply from Earth. Acta Astronautica, 1992, 26, 295.

15. Paprotny, Z., Signals from ETI detected - what next? Acta Astronautica, 1990, 21, 93.

16. Agreement governing the activities of states on the Moon and Other celestial bodies, art. 5(3), entered into force 11 July, 1984, 1363 U.N.T.S. 3, text reproduced in Report, Committee on the Peaceful Uses of Outer Space, 34 U.N. GAOR Supp. (No. 20), U.N. Doc. A/AC.105/L.113 add 4, 1979, United Nations Treaties on outer space 22, 1999, and 18 I.L.M. 1434, 1979.

17. Treaty on principles governing the activities of states in the exploration and use of outer space, including the Moon and other celestial bodies, art. XI, opened for signature 27 January, 1967, 18 U.S.T. 2410 T.I.A.S. No. 6347, 610 U.N.T.S. 205.

18. ITU footnote (722), Radio Regulation 8-94, \& Report 700-1 on Radiocommunication Requirements for Systems to SETI, refer to the use of the radio frequency spectrum for the SETI, and in particular, in the $1400-1727 \mathrm{MHz}, 101-120 \mathrm{GHz}$ and $197-220 \mathrm{GHz}$ bandwidths. Kopal, V. International law implications of extraterrestrial intelligent signals. Acta Astronautica, 1990, 21, 123. The first of these bandwidths relate to the so-called "watering-hole" between the hydrogen line at $1420 \mathrm{MHz}$ and the hydroxyl line at $1662 \mathrm{MHz}$. Note by the Secretariat, COPUOS, Messages to Extraterrestrial Civilizations, para. 13, U.N. Doc. A/AC.105/206, 18 October, 1977.

19. Reijnen, G. C. M., Basic elements of an international terrestrial reply following the detection of a signal from extraterrestrial intelligence. Acta Astronautica, 1990, 21, 143,147.

20. Reijnen, G. C. M., Legal aspects of outer space, Chap. VIII. Extraterrestrial intelligence and Earthian law, Thesis, Utrecht University, p. 179. 1976.

21. Cocca, A. A., XII tables for researchers on extraterrestrial intelligence. Acta Astronautica, 1990, 21, 127.

22. Haley, A., Space Law and Government, 1963, p. 413.

23. Körner, S., Laws of thought. In The Encyclopedia of Philosophy, vol. 4, ed. P. Edwards. Macmillan Publishing Co. Inc, 1972, pp. 416-417.

24. Durant, W., This is a situation which would have been found by an ETI contacting human civilization in the not so distant past. In The Story of Civilization, our Oriental Heritage, vol. 1. Simon and Schuster, 1954, pp. 19-20. 